\title{
ARTICULACIÓN ESTRUCTURAL EN EL DISTRITO FEDERAL
}

\section{STRUCTURAL ARTICULATION IN MEXICO CITY}

\begin{tabular}{c|c} 
Alonso Jiménez Reyes & Marco Antonio Márquez Mendoza \\
Universidad Nacional & Universidad Nacional \\
Autónoma de México & Autónoma de México \\
seisreyes@hotmail.com & antoniomrqz@gmail.com
\end{tabular}

\section{RESUMEN}

La articulación estructural se refiere a la forma en que los agentes económicos interactúan en la compra y venta de bienes y servicios. Desde la estructura productiva, las industrias son oferentes y demandantes de insumos y productos, cuyas interrelaciones forman redes, en las que cada industria juega un papel distinto. A partir de las redes económicas es posible identificar el papel articulador de cada industria, es decir, si emite o recibe influencia de los miembros de la red, o si tiene vínculos fuertes con otros, así estas características se refieren a la centralidad que tiene un agente en la estructura. Este trabajo utiliza la estimación de una tabla de insumo producto para la economía del Distrito Federal del 2008 cuyo interés es identificar los llamados Coeficientes Importantes que determinan el nivel de integración y por medio de los cuales se generan grafos dirigidos y a partir de estos resultados se identifica la forma de articulación mediante las medidas de centralidad propuestas por las redes sociales pero medidas desde la teoría de grafos. 


\title{
Palabras clave: Modelo de insumo - producto, integración, redes eco- nómicas, teoría de grafos
}

JEL: C02, C67, F15

\begin{abstract}
The structural articulation refers to the way in which economic agents interact in the buying and selling of goods and services. From the structure of production, industries are buyers and sellers of inputs and outputs, whose interrelations are networks in which each industry plays a different role. From economic networks can identify the coordinating role of each industry, ie, whether sending or receiving influence of network members, or have strong links with others, and these characteristics refer the centrality that has an agent in the structure. This paper uses the estimation of a input output table to the economy of Mexico City in 2008, the interest is to identify the most important coefficients that determine the level of integration and through which directed graphs are generated and from these results is identified the centrality measures proposed by social networks but measures from graph theory
\end{abstract}

Keywords: input output model, integration, economic networks, graph theory 


\section{INTRODUCCIÓN}

La articulación estructural se refiere a la forma en que los agentes económicos interactúan en la compra-venta de bienes y servicios. Desde la esfera productiva, las industrias son oferentes y demandantes de insumos y productos cuyas interrelaciones forman redes económicas regidas por las fuerzas de mercado, no obstante, los estímulos de la demanda final hacen que cada sector se articule de manera distinta en la red económica. La actuación de cada agente es mediada por el Estado ya que legaliza e institucionaliza las normas de acción. Sin embargo, el papel de cada agente es acorde con el contexto económico, ya que en épocas de recesión las decisiones de los agentes son distintas que en épocas de auge.

La articulación estructural puede ser determinada por la intervención y la no intervención económica del Estado en los mercados nacionales e internacionales. En el caso de México, desde 1936 hasta 1982 la intervención económica del Estado dirigió un tipo de integración en la que los sectores productivos demandaban mayores insumos nacionales debido a los altos aranceles en algunos mercados. La integración se dio dentro de la economía ya que el Estado garantizaba mercado e insumos, lo que hizo empresas poco competitivas en el país. Así, en la época de no intervención económica, se liberalizó la economía y las empresas no competitivas fueron cerradas al no poder enfrentar la competencia exterior. De esta manera, la integración económica de México en la actualidad está orientada al exterior, donde se busca una mejor inserción a las cadenas de valor globales (Minian y Dávalos; 2012).

Desde la década de los ochenta la integración económica ha sido la incorporación de los sectores económicos a las cadenas productivas globales de las industrias, como la automotriz, la química, la farmacéutica, la electrónica, la maquinaria y equipo, entre algunas. No obstante, la integración hacia el exterior ha hecho a la economía más vulnerable y su desempeño ha sido muy cuestionado.

Nacionalmente se ha mostrado que la integración económica al exterior no ha producido los multiplicadores del comercio y el impacto favorable en el uso de factores, como lo proponía la teoría del comercio internacional (Krueger, 1990). Lo que ha sucedido en la economía 
mexicana ha sido una integración al exterior por medio de las importaciones de insumos, aunque el sector exportador ha sido dinámico hay un problema de encadenamientos hacia atrás en las exportaciones y en la economía nacional (Ruiz y Moreno, 2006)

En el caso de la economía del Distrito Federal (DF), la estrategia nacional ha hecho de ella una economía de servicios, tal como lo muestran los datos del Instituto Nacional de Estadística y Geografía, INEGI. De hecho, la economía nacional ha dado mayor peso al sector servicios a costa de una reducción del sector primario. A pesar de que el DF tiene la política de no intervención económica, el gobierno de la zona se ha caracterizado desde su creación como un gobierno de izquierda y ha sido la entidad más representativa en indicadores sociales, como el índice de desarrollo humano, el índice de educación, salud e ingreso (PNUD, 2010).

Desde el modelo de insumo-producto (MIP) es posible captar las interrelaciones que tienen los sectores y medir la forma de articulación que hay entre ellos, lo cual refleja el desarrollo que tiene una economía, además de que el MIP permite hacer un análisis de la senda que sigue un impulso de la oferta o demanda sobre la estructura económica. Los sectores transmiten sus impulsos en las redes, en los canales de comercialización o al inventario de proveedores, ya que las actividades de las industrias son de una perspectiva doble, son oferentes de productos y demandantes de insumos.

La intensidad de las relaciones desde el MIP ha sido captada desde la teoría de redes, las cuales subrayan los beneficios de una red perfectamente integrada. En este trabajo se identifican las redes económicas del DF y se miden algunos indicadores de centralidad, como la densidad, cercanía e intermediación. Estos indicadores no son para redes valuadas como en algunos trabajos de redes sociales proponen (Borgatti, 2005), sino más bien se miden a partir de la propuesta de grafos no valuados, es decir, para datos binarios, así el trabajo destaca el hecho de que dichos indicadores son un reflejo del grado de articulación económica que hay en la zona.

Para tales fines el estudio, despues de la introducción, está organizado en tres secciones, en la primera se explican los vínculos de la metodología de insumo producto y la teoría de redes, en esta sección se incluye un apartado que define el modo de articulación desde las medidas de 
centralidad propuestas por las redes sociales, pero medidas desde la teoría de grafos. En el segundo apartado se analiza la economía del DF mediante la tabla de insumo-producto para dicha entidad (Jiménez, 2013) y refleja la forma de articulación mediante la centralidad de las redes económicas para datos binarios. Finalmente, se considera un apartado de conclusiones.

\section{LA TEORÍA DE REDES ECONÓMICAS}

Un sistema económico se constituye por un conjunto de agentes económicos que se interrelaciona por medio de flujos de bienes y servicios. Los agentes económicos juegan dos papeles en este flujo, son oferentes y demandantes en el mercado. Así, por ejemplo, las familias demandan bienes y servicios de consumo final de las industrias y del gobierno, y ofrecen mano de obra a estos mismos. La estructura, por su parte, es un subconjunto de dicho sistema, ya que el objeto de estudio es la interrelación que tienen las industrias o sectores, ellas demandan bienes para emplearlos como insumos en la producción y, a su vez, ofrecen bienes que se emplean como insumos o para consumo final.

Por medio de estos flujos de demanda y oferta los sectores se influencian unos a otros, por ejemplo, los compradores de bienes (que emplearán como insumos) influyen en la determinación del nivel de producción de otros, ya que un incremento de la demanda de estos implica un crecimiento del producto de otros, de tal forma que la compra de bienes de una industria a otra influye en las decisiones sobre inversión, gasto y nivel de empleo de otras industrias (Leontief, 1986). Como vendedores de bienes también influyen en la producción, por ejemplo, al incrementar su oferta por efecto de una innovación pueden estimular el uso de la innovación en otros sectores, y así estar en posibilidades de incrementar su oferta.

Las influencias intersectoriales se clasifican entonces por el sentido que adoptan, si provienen desde la demanda se definen como encadenamientos o vínculos hacia atrás o de arrastre y si provienen de la oferta, son encadenamientos o vínculos hacia adelante o de empuje. Los sectores entonces pueden clasificarse también por su capacidad para influir sobre otros productores y sobre el sistema económico como un todo. Así, en una 
economía integrada, las influencias sectoriales implican un gran número de conexiones entre las industrias por efecto de la compra y venta de insumos o productos.

En la articulación de la estructura productiva las industrias complementan sus procesos productivos unas con otras y, ante un cambio de la demanda final o del valor agregado, se propagan de manera distinta en los miembros de la estructura, pues la evidencia muestra que no todos los sectores tienen el mismo papel como impulsores del crecimiento y que la integración y, por tanto, su articulación, en su estructura, no es homogénea.

Como es sabido, la teoría de grafos ha desarrollado sus propios conceptos y métodos de acuerdo con la existencia de un grupo de nodos (vértices, agentes, industrias) y de las relaciones existentes en dicho grupo (aristas, arcos, vínculos, conexiones), que matemáticamente hace consistente a cualquier tipo de red. Desde este análisis, el objeto de estudio son las relaciones y los agentes; el cual es el mismo objeto de estudio del MIP. De hecho, se han desarrollo algunos algoritmos para determinar propiedades de las relaciones de la red, esto ha sido definido como el enfoque pretopológico.

Sin embargo, algunos autores consideran que, a pesar de que hay conceptos válidos, es decir son topológicamente coherentes, no todos los métodos de transformación de la matriz de coeficientes a una binaria tienen consistencia, como el caso del análisis de flujo mínimo propuesto por Schnabl (1995), que busca un subconjunto de (I-A) ${ }^{-1}$, es decir, una matriz $\left(\mathrm{A}^{\mathrm{n}}\right)$ la cual muestra influencias cada vez menores dentro del sistema, esto es, muestra como la difusión de un efecto sobre las redes económicas toma caminos cada vez más débiles de acuerdo con las influencias indirectas de los estratos de la matriz de Leontief. De Mesnard (1995) enfatizó que dicha metodología implica no transitividad al utilizar un filtro para cada estrato en el análisis del MIP.

De forma general, si la estructura económica es un grupo de industrias que están interconectadas por las compras y ventas de insumos y productos, entonces dicha estructura puede ser considerada como un grafo o una red, donde los nodos son las industrias y las conexiones son los flujos de comercio. Si atendemos el flujo de comercio, entonces podemos analizar dichos vínculos por el lado de la oferta o de la demanda, por consiguiente las redes económicas son digrafos. 
Para analizar la topología entre el MIP y la teoría de redes es necesario establecer que son conjuntos preordenados. Si se define a $A$ como la matriz de coeficientes técnicos y $W$ como una matriz de adyacencias asociadas a la matriz $A$, de acuerdo con el MIP se sigue que $(I-A)^{-1}=I+A^{1}+$ $A^{2}+A^{3} \ldots .+A^{\infty}=\sum_{i=0}^{\infty} A^{\infty}$, es decir, son las influencias directas e indirectas que hay entre los sectores productivos, así el estrato de la segunda ronda $\left(A^{2}\right)$ muestra un mayor grado de influencia que en el caso de un estrato más lejano en este proceso iterativo, por ejemplo $A^{5}$.

Una aproximación de este concepto es el número de caminos (walks) con longitud $n$, es decir el número de caminos que hay del sector $i$ al sector $j$, con longitud $n$, donde las longitudes son las conexiones (Harary,1969). Esto implica que no importa si algunas conexiones y, por ende, algunos sectores se repiten para llegar al destino, por ejemplo, si queremos encontrar el camino del sector $i$ al $j$ es posible que tengamos $i \rightarrow k \rightarrow l \rightarrow m \rightarrow k \rightarrow j$ dentro del grafo, por lo que $\sum_{i=1}^{\infty} W^{\infty}$ es el número de caminos y no sendas (ya que en el caso de las sendas los vértices y las conexiones son únicas).

Es posible notar que se trata sólo de un concepto, que en el nivel abstracto son parecidos, sin embargo, pretopológicamente distintos, ya que $W^{0}$ no existe en el número de caminos que hay en el grafo. Así $W$ no es reflexiva como en el caso de $A$. Muchos autores que han empleado el análisis cualitativo lo hacen sin bucles, es decir, utilizado la diagonal principal con cero (Aroche y Márquez, 2011; García, Aroche y Ramos, 2007), esto hace que la matriz de coeficientes técnicos, la de multiplicadores y la matriz de adyacencias no sean reflexivas.

Tanto $\sum_{k=1}^{\infty} W^{\infty}$ como $\sum_{k=0}^{\infty} W^{\infty}$ puede guardar un preorden $(\succsim)$ de tal forma que si $A^{1} \succsim A^{2} \gtrsim A^{3} \ldots \succsim A^{\infty}$ entonces $A^{1} \succsim A^{\infty}$, por lo que si $W^{1} \succsim W^{2}$ $\gtrsim W^{3} \ldots \succsim W^{\infty}$ entonces $W^{1} \succsim W^{\infty}$, es decir, ambas son racionales. Como es sabido $\sum_{i=0}^{\infty} A^{\infty}$ es la unión de conjuntos ordenados $(\geq)$, de esta manera $A^{1}$ $>A^{2}>A^{3} \ldots A^{\infty}$, por lo que $A^{1}>A^{\infty}$, en este sentido si $A \rightarrow W$ entonces debería ser que $W^{1}>W^{2}>W^{3} \ldots W^{\infty}$ y $W^{1}>W^{\infty}$, no obstante, se ha mostrado que las entradas de $W^{\infty}$ tienden a infinito (Hage y Harary, 1991), por lo que $\sum_{i=0}^{\infty} A^{\infty} \nrightarrow \sum_{i=1}^{\infty} W^{\infty}$.

Esta variante puede dejar de tener sentido si enfatizamos el concepto de cada una de las matrices, así $(I-A)^{-1}$ puede ser desglosada a partir 
de los estratos, los cuales son cada vez más pequeños, la agregación de estos estratos hacen el total de relaciones directas e indirectas en la estructura, así, aunque ordenadamente, $A^{\infty}$ tiende a cero (Miller y Blair, 2009) y $W^{\infty}$ tiende a infinito, cualitativamente son las mismas, se trata del total de relaciones directas e indirectas, es decir, del camino directo e indirecto de la transmisión de la influencia del sector $i$ al $j$.

El análisis cualitativo del MIP busca identificar el esqueleto de la estructura, por ello es la utilización del filtro $(\varphi)$, ya que si $a_{i j} \in A$ y $w_{i j} \in W$ y si $A \rightarrow W \leftrightarrow \phi>0$ entonces si $a_{i j}>0 \rightarrow w_{i j}=1$ y si $a_{i j}=0 \rightarrow w_{i j}=0 \mathrm{se}$ identificaría el total de vínculos existentes en la estructura, ya que tanto la matriz $A$ como $W$ son de doble entrada; sin embargo, no todos los vínculos son relevantes para identificar el esqueleto. Es por eso que los trabajos que recurren a la teoría de redes emplean un filtro $(\varphi)$ que permita analizar una subestructura que refleje las relaciones importantes del flujo económico. En este trabajo se emplean los coeficientes importantes (CI) como una medida de integración de la estructura económica, ya que identifica aquellas entradas que cuando cambian provocan mucho más influencias directas e indirectas que el resto de los miembros de la estructura económica.

\subsection{Articulación y centralidad en redes}

La búsqueda de los sectores clave fue una tarea que nació con la tesis del crecimiento desbalanceado de Hirschman (1958) y su hipótesis de la existencia de sectores en la economía que tienen efectos diferenciados por el lado de la oferta o demanda. Estos sectores denominados claves fueron identificados en diversos trabajos y representan el nivel de articulación de la economía. No obstante, la articulación económica en el MIP también se refiere a la dependencia que hay entre los procesos productivos de las diferentes industrias, un cambio en el tamaño de la demanda de un insumo provoca cambios en las demandas de otros, si el cambio en demanda se debe, por ejemplo, a cambio en la utilización de factores. Que desde el MIP se explica por un cambio en la proporción de insumos utilizados definidos como coeficientes técnicos.

La literatura de los coeficientes importantes parte de los resultados de trabajos de la década de los cincuenta del siglo pasado, en los que se 
demuestra que para una matriz no singular y su inversa, si uno o más elementos de la matriz cambian se producen cambios en la inversa de uno o más elementos, ajustándose algunos otros de toda la estructura; tanto para el caso del cambio en un solo elemento (Scherman y Morrison,1949) como para el de varios elementos de manera simultánea (Woodburry; 1950). Por lo que se modifica la articulación entre los sectores al modificarse las interrelaciones por renglón y columna, es decir, como oferente y demandante. Si cambia el insumo de un sector el nivel de producto cambia y en el proceso iterativo con otros sectores modifica también el nivel del producto de aquellos sectores. Si el cambio de insumos impacta positivamente sobre el producto, cuanto más influencias genere el cambio de insumos sobre otros sectores cuanto mayor articulador es sobre la estructura económica. De acuerdo con Shintke y Stäglin (1988) los CI se identifican por el análisis de sensibilidad mediante los límites de tolerancia como:

$$
r_{i j}=\frac{1}{a_{i j}\left[\propto_{j i}\left\{\left(\propto_{i i} / x_{i}\right) x_{j}\right\}\right]}
$$

donde $a_{i j}$ son elementos de la matriz $A, \propto_{j i}$ son elementos de la matriz inversa de Leontief $L, x$ es el valor bruto de la producción. Así un sector central en la estructura tiene mayores entradas importantes que el resto de los miembros. Las entradas importantes muestran los vínculos que tiene mayor relevancia.

Desde la teoría de redes sociales la integración ha sido tratada como la identificación del agente central de un grupo, el cual se define como aquel que tiene mayor influencia en los demás miembros del grupo, es decir, es el agente que tiene más vínculos con los miembros de forma directa (Shaw,1954), el agente central determina el modo de organización de un grupo y la forma de resolver un problema, se convierte en una especie de líder en un grupo social (Cohen,1964, Freeman,1979).

En la literatura hay diversos modelos sobre el concepto de centralidad, por ejemplo, algunos autores han asociado el concepto de centralidad con la rapidez en el flujo de una variable dentro de la red social (Borgatti, 2005). No obstante han estado de acuerdo con que el concepto de centralidad tiene que ver, por lo menos, con tres elementos (Freeman,1979; Newman, 2005). 
La densidad es la primera medida de centralidad y se refiere al número de influencias que emite o recibe un agente social en la estructura, en este sentido la densidad de un grupo es la complejidad de conexiones directas e indirectas que guardan los miembros de la red. Una forma habitual de calcular dicha media es a partir de la agregación de las adyacencias, sean de recepción (indegree) o de emisión (outdegree):

$$
\begin{aligned}
& \Delta=k\left(n^{2}-n\right)^{-1} \\
& \kappa=\iota W \iota^{\prime}
\end{aligned}
$$

$\imath$ es un vector columna, W es la matriz de adyacencia con diagonal principal igual a cero, $n$ es el número de elementos de la estructura, $\kappa$ es un escalar que suma las entradas positivas de $\mathrm{W}$, las cuales son el total de los arcos del grafo. Como en los trabajos convencionales se ha utilizado W irreflexiva, entonces $\left(n^{2}-n\right)$ muestra el escalar máximo de posibles arcos en un grafo completo. Si se trata de un grafo perfectamente conectado y reflexivo, habría un total de arcos de $\mathrm{n}^{2}$, por lo que la densidad pudiera considerarse como $\left(\iota W \iota^{\prime}\right)\left(n^{2}\right)^{-1}$, así si un grafo es completo el grado de complejidad máximo que alcanza es 1 en ambos casos.

La densidad puede ser considerada por la dirección que tengan los vínculos, por efecto de recibos o de envíos de influencia, se ha demostrado que la transpuesta de una matriz de adyacencias implica el cambio de dirección de las influencias, se conserva la estructura, por lo que el grado de densidad total para un grafo es el mismo, no obstante, el grado de complejidad que tienen los agentes no necesariamente es el mismo en el proceso de influencias, pero tampoco lo es para el caso de las redes que surgen por el lado de la oferta, como aquellas que se originan en la demanda.

Si se analizan aquellas redes que surgen por el lado de la demanda o por la oferta, tanto la recepción y la emisión de influencias para cada sector varía. La siguiente ecuación expresa el grado de complejidad alcanzado en la recepción $\left(D^{i}\right)$ y emisión $\left(D^{o}\right)$ de influencias en cada sector.

$$
\begin{aligned}
& D^{i}=\iota W(n-1)^{-1} \\
& D^{o}=W \iota^{\prime}(n-1)^{-1}
\end{aligned}
$$


Esta medida de centralidad implica que el grado nodal para cada sector es representativo para el nivel de integración del agente en la estructura. Así, entre más densa sea una red, es decir, su grado de complejidad se aproxime a 1, mayor será la integración de la estructura. Esta medida de centralidad también ha sido considerada para los grafos evaluados; sin embargo, tanto la cercanía como la intermediación, aunque sean conceptos propuestos por las redes sociales, en economía toman un matiz más particular, más aún cuando dicha medición se hace con datos binarios, que, a diferencia de las redes sociales, se han propuesto para grafos valuados (Borgatti, 2005).

Desde las redes sociales se ha discutido el concepto de cercanía, el cual hace referencia a la distancia que hay entre un punto y otro, si y sólo si este camino es una senda. Así, la geodésica senda de un punto $a_{i}$ a otro $a_{j}$ es capturada en una matriz D con elementos $d_{i j}$; si no hay senda entre $a_{i}$ y $a_{j}$ entonces es infinito, mientras que si $d_{i j}$ es diferente a cero las entradas $d_{i j}$ muestran las sendas mínimas de longitud $k$ que conectan a un sector con otro (Harary, 1969). Como se discutió en el apartado anterior, la matriz $\sum_{k=0}^{\infty} W \#^{\infty}$ es la matriz de accesos que muestra la conexión entre dos nodos de acuerdo con el tamaño de longitud, así podemos definir la matriz de sendas cortas de longitud k como:

$\sum_{k=0}^{n} W^{n} \#=\left(I \#+W \#+W \#^{2}+W \#^{3}+\cdots+W \#^{n}\right)$

No obstante, el concepto de cercanía en las redes económicas parte del problema de que la senda de $a_{i}$ a $a_{j}$ no es igual a la senda de $a_{j}$ a $a_{i}$, esto implica que un sector mantiene relaciones cercanas con otros si y sólo si la senda $a_{i}$ a $a_{j}$ es igual a la de $a_{j}$ a $a_{i}$. Sin embargo, si es la distancia más corta en redes no dirigidas es de longitud 1; para los digrafos, como es el caso de las redes económicas, la geodésica distancia entre $a_{i}$ y $a_{j}$ es de longitud 2. En este sentido, la integración de los procesos productivos implicaría que un sector tiene vínculos por la demanda con otro sector, y este último también le demanda insumos al primer sector. Es decir que, por efecto de la demanda, dos sectores tienen relaciones recíprocas, pero esto igual se puede pensar por el lado de la oferta. 
Si una matriz de adyacencia es simétrica implica que sus elementos mantienen relaciones recíprocas. Sin embargo, en las redes económicas no todos los sectores pueden mantener relaciones reciprocas, por ejemplo, es posible que la industria de la silvicultura demande productos de la industria de la electrónica, pero no al revés. Por otro lado, también es posible que la industria farmacéutica demande productos de la industria de la agricultura y que la agricultura demande productos de la industria farmacéutica.

En el supuesto de que las industrias pudieran mantener relaciones recíprocas, una red perfectamente integrada implicaría la máxima cercanía. Si un dígrafo es completo, la matriz de adyacencias es una matriz de unos, si definimos la cercanía como la geodésica senda del punto $a_{i}$ a $a_{j}$ es igual a la de $a_{j}$ a $a_{i}$ entonces es de longitud 2, por lo que la matriz de reciprocidad perfecta $\left(W_{R}^{*}\right)$ es una matriz con elementos igual a 2 .

Ahora bien, para medir la cercanía del sector en la estructura definimos la matriz de reciprocidad $\left(W_{R}\right)$, cuyos elementos son $\hat{a}_{i j}=a_{i j}+a_{j i}$ así tenemos que $i W_{R}=W_{R} i^{\prime}$. Entonces el índice de cercanía económica de longitud 2 es igual a:

$$
W_{C E}=\left(W_{R} \iota^{\prime}\right)\left(W_{R}^{*} \iota^{\prime}\right)^{-1}
$$

las entradas del vector $W_{\mathrm{CE}}$ tienden a 1 si son cercanas; si es igual a 1 , un sector demanda u ofrece con el resto recíprocamente, tiende a cero si hay alejamiento, si fuera cero implicaría que el sector no tiene senda con la estructura económica, es un sector aislado.

Finalmente, una tercera medida sobre centralidad es la intermediación; esta ha sido trabajada por las redes sociales y los modelos típicos que la han utilizado en los modelos del flujo de información (Nieminen, 1973), es decir, se trata de una medida de quien controla o quien permite el acceso $a$. Si de $a_{i}$ a $a_{j}$ es una senda, existe un $a_{l}$ que es un intermediario entre $a_{i}$ y $a_{j}$, si $a_{l}$ es el intermediario más corto entre $a_{i}$ y $a_{j}$ es porque la senda de $a_{i}$ a $a_{j}$ es de longitud 2. Así, un nodo cuando está conectado con el resto de la estructura puede ser un buen intermediario, en el sentido de que es posible acceder al resto de los agentes por medio de él. El total de intermediaciones de un sector puede ir creciendo si se consideran las 
posibles sendas mayores a 2. El siguiente algoritmo resuelve el grado de intermediación que tiene un sector en la senda de longitud 2.

Si $a_{l}$ es un elemento de un grafo y este emite influencia al resto de la estructura, entonces es el intermediario mínimo de aquellos elementos de los que recibe influencia el elemento $a_{l}$. Por lo que puede servir de intermediario de longitud 2 a otro elemento en (n-2) donde $n$ es el número de elementos. En efecto, es menos 2 debido a que $a_{l}$ es intermediario de un $a_{i}$ con $a_{j}$. Si $a_{l}$ recibe de todos influencia entonces $a_{l}$ puede ser intermediario en (n-1). Se trata de dos conceptos, intermediado en (n-2) y de intermediador de (n-1). Así, en el caso de que un agente reciba y emita influencia a todos los agentes de la estructura, el total de veces que podrá ser intermediario de una senda $a_{i}$ a $a_{j}$ de longitud 2 es:

$$
T_{2_{l}}^{i n t}=(n-2)(n-1)
$$

$T_{2_{l}}^{\text {int }}$ es un escalar que mide el total de intermediaciones de longitud 2 del elemento $l$. Una forma de calcular el grado de intermediación de longitud 2 para cada agente es tomar el reglón del agente y su columna, el renglón indica los sectores a los que envía influencia y la columna indica la influencia que recibe de otros sectores.

El grado de intermediación depende del tamaño de influencias que envíe un sector y del tamaño de influencias que recibe dicho sector. En una matriz de adyacencia $\mathrm{W}$, los renglones indican la transmisión de influencias y las columnas el recibo de influencias, por lo que la intermediación de un sector dependerá del vector renglón del sector $l\left(\ddot{a}_{i}\right)$ y de su vector columna $\left(\ddot{a}_{i}{ }^{\prime}\right)$. Para encontrar el total de intermediaciones que tiene cada sector podemos definir la matriz de intermediaciones como:

$$
W \iota=W+W^{\prime}
$$

Donde $\mathrm{W}$ es la matriz de adyacencias con diagonal cero. Las operaciones de esta matriz es elemento a elemento y tienen la siguiente característica:

$1+1=1$, $0+1=1$, 
$0+0=0 \mathrm{y}$

$1+0=0$,

si las entradas de $W \iota$ son 0 , si $a_{l}$ no es intermediario para que se conecte $a_{i}$ con $a_{j}$, ya que, en ambos casos, no se reciben conexiones por $a_{i}$, mientras que si son 1 es posible que $a_{l}$ sea intermediario para que se conecte $a_{i}$ con $a_{j}$. Sin embargo, hay que diferenciar que significa $1+1=1$ y $0+1=1$, para el primer caso el total de intermediaciones es el total de conexiones menos 1 , dado que son recíprocas, por lo que $a_{l}$ emite influencias a $a_{j}$ y al revés, porque es un camino y no una senda, las intermediaciones que tiene se le restan los envíos que se hacen a $a_{i}$. Por otro lado, en el caso de el segundo termino $(0+1=1)$ implica que $a_{i}$ se conecta con $a_{l}$ para acceder a $a_{j}$, que, al no considerarse la reciprocidad, el total de intermediaciones es el total de envíos. En esta matriz los elementos de la diagonal principal son cero, ya que un sector no es intermediario para si mismo.

Así, el total de intermediaciones de un sector $l$ en toda la estructura es igual a la suma de las intermediaciones que tiene cada sector $i$ con el sector $j$ por medio del sector $l$, las cuales son de longitud 2 y dependen del total de influencias emitidas. En este sentido, las entradas de tienen que ser sustituidas por el total de envíos de acuerdo con los criterios de las operaciones en la estructura. El grado de intermediación de longitud 2 para el sector 1 es:

$$
\mathrm{I}_{i}^{\circ}=I T_{i} / T_{2_{i}}^{i n t}
$$

donde, $I T_{i}$ representa el total de intermediaciones que genera el sector i y $T_{2_{i}}^{\text {int }}$ es el total de intermediaciones que pudiera tener el sector i en toda la estructura. De igual forma, un sector que es el máximo intermediario tendría $100 \%$ de integración o 1 , de acuerdo con la ecuación (9) ${ }^{1}$.

\footnotetext{
${ }^{1}$ Este procedimiento no es para la intermediación de grado $\mathrm{n}$ como lo desarrollan algunos software, ya que la intermediación ha sido para datos valuados, por lo que se encuentran, por ejemplo, con el índice de Kendall.
} 


\section{LAS REDES ECONÓMICAS EN EL DF}

En esta sección se comenzará describiendo, a grandes rasgos, la construcción de la tabla de insumo producto para el caso de la economía del DF (sus detalles pueden consultarse en Jiménez, 2013).

En primer lugar se utilizaron los datos de los censos económicos capturados por el INEGI, referidos al consumo intermedio, la demanda intermedia y el valor agregado. Dichos datos están a precios constantes de 2003 y se refieren al año 2008. Se consideró también el tamaño del producto de la entidad con año base de 2003, para el mismo año, y a precios básicos como se utiliza en la TIP nacional de 2003.

Dado que la actividad económica del DF tiene una misma dinámica que el crecimiento de la economía nacional, además de que su aportación al nivel de producto es mayor que el del resto de las entidades federativas, la tabla se construyó con el supuesto de economía representativa, el cual dentro del MIP implica que las relaciones que hay entre los sectores de la economía del DF sigue patrones parecidos a los de la economía nacional.

Una vez considerado este supuesto, se desarrolló el algoritmo de RAS que, desde la metodología del MIP, se utiliza para estimar la actualización de las tablas de insumo-producto, con las consideraciones de que el producto es un equilibrio contable en el que la suma renglón no desequilibra la suma columna, esto es, que la generación del consumo intermedio no descompensa a la generación de la demanda intermedia y que, aun siendo distintos, ambas variables se compensan; con el valor agregado por el lado de la oferta y con la demanda final por el lado de la demanda. Bajo el supuesto de economía representativa se actualizaron los datos de la tabla de transacciones interindustriales nacional, pero cuyas restricciones fueron los datos de la economía del DF para el año 2008 a precios de 2003. Así, las bondades de la tabla son dos: mantiene los precios de las transacciones de 2003 y es comparable al de la economía nacional, por ello dicha tabla es una subestructura de la economía nacional.

\subsection{Resultados}

El cuadro 1 muestra los límites de tolerancia para la estructura económica del DF. Dicho cuadro refleja cambios que son de menos infinito a 
más infinito. De manera convencional, se utilizan los cambios que van de 0 a 20\% como límite superior, es decir, una modificación de, al menos, 20\% en los coeficientes técnicos. Bajo tal criterio, el porcentaje de entradas importantes de la estructura económica del DF es de $8.5 \%$, esto es, 34 vínculos importantes de 400 que se tienen en la matriz (el orden de la matriz es de 20). No obstante, de acuerdo con estos resultados es posible visualizar la senda de influencias que siguen los sectores ante cambios en las entradas $a_{i j}$ en la estructura. El grafo 1 muestra la red económica del DF, como se aprecia existen líneas gruesas que indican las relaciones recíprocas entre los miembros, mientras que las otras líneas son relaciones de emisión o transmisión de influencias en la estructura económica.

De esta manera es posible diagnosticar cual es el camino de influencias que siguen los sectores económicos, por ejemplo, un cambio en la demanda de insumos en el sector transporte influye fuertemente en los sectores de electricidad y agua, en la dirección de corporativos, en los servicios a la educación y los servicios de gobierno, no obstante, una vez que cada uno de estos sectores hace el cambio correspondiente van generando influencia a otros sectores, por ejemplo, la influencia del cambio en el transporte modifica la demanda de insumos del Gobierno y, junto a él, modifica las relaciones de compra de otros sectores como son la construcción, la electricidad, otros servicios excepto actividades del gobierno e incluso el mismo sector transporte, ya que este sector guarda una relación reciproca con el de actividades de gobierno.

\section{Cuadro 1}

Entradas importantes de acuerdo con el índice de sensibilidad

\begin{tabular}{|c|c|c|c|}
\hline \multicolumn{2}{|c|}{ Limites Tolerables } & \multicolumn{2}{|c|}{ Frecuencia } \\
\hline Inferior & Superior & Relativa & Acumulada \\
\hline$-\infty$ & 0 & 0.21 & 82 \\
\hline$>0$ & $<=5$ & 0.23 & 92 \\
\hline$>5$ & $<=10$ & 0.26 & 105 \\
\hline$>10$ & $<=20$ & 0.29 & 116 \\
\hline$>20$ & $<=50$ & 0.39 & 155 \\
\hline$>50$ & $<=100$ & 0.48 & 193 \\
\hline$>100$ & $+\infty$ & 1.00 & 400 \\
\hline
\end{tabular}

Fuente: Elaboración propia. 


\section{Gráfico 1}

Las redes económicas en el DF

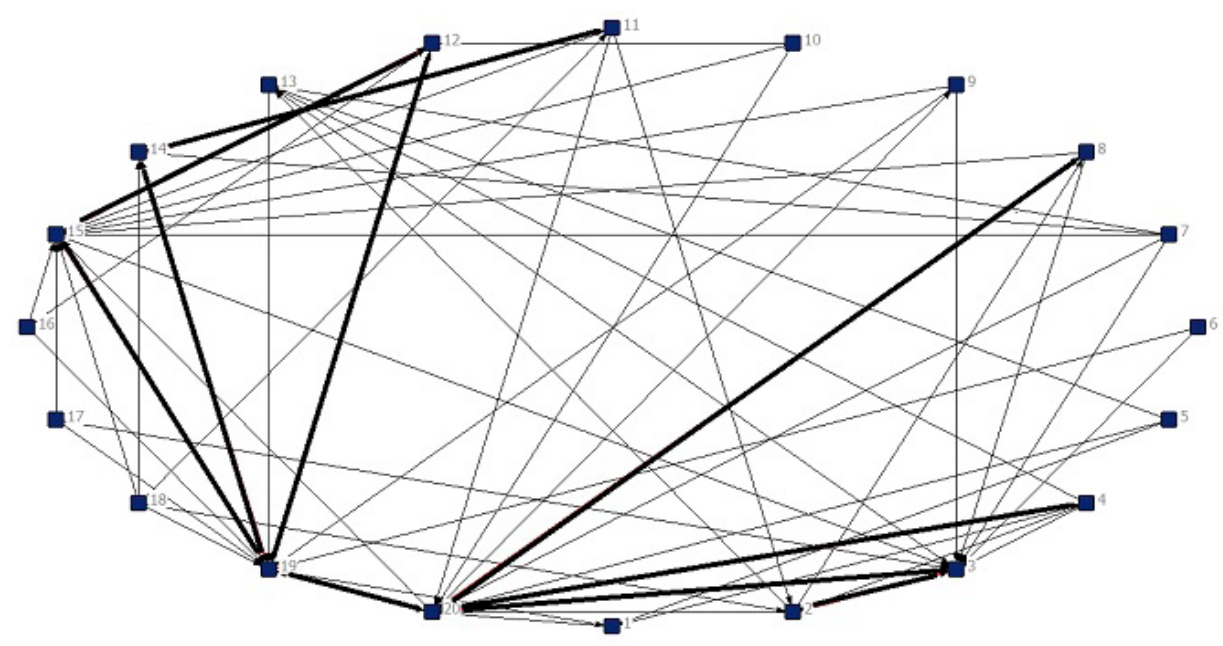

\begin{tabular}{|r|l|r|l|}
\hline \multicolumn{3}{|c|}{ Sectores económicos } \\
\hline 1 & $\begin{array}{l}\text { Agricultura, ganadería, aprovechamiento } \\
\text { forestal, pesca y caza }\end{array}$ & 11 & $\begin{array}{l}\text { Servicios inmobiliarios y de alquiler de } \\
\text { bienes muebles e intangibles }\end{array}$ \\
\hline 2 & Minería & 12 & $\begin{array}{l}\text { Servicios profesionales, científicos } \\
\text { y técnicos }\end{array}$ \\
\hline 3 & $\begin{array}{l}\text { Electricidad, agua y suministro de gas } \\
\text { por ductos al consumidor final }\end{array}$ & 13 & Dirección de corporativos y empresas \\
\hline 4 & Construcción & 14 & $\begin{array}{l}\text { Servicios de apoyo a los negocios y mane- } \\
\text { jo de desechos y servicios de remediación }\end{array}$ \\
\hline 5 & Industrias manufactureras & 15 & Servicios educativos \\
\hline 6 & Comercio & 16 & Servicios de salud y de asistencia social \\
\hline 7 & Transportes & 17 & $\begin{array}{l}\text { Servicios de esparcimiento culturales y } \\
\text { deportivos, y otros servicios recreativos }\end{array}$ \\
\hline 8 & Transportes y almacenamiento & 18 & $\begin{array}{l}\text { Servicios de alojamiento temporal y de } \\
\text { preparación de alimentos y bebidas }\end{array}$ \\
\hline 9 & Información en medios masivos & 19 & $\begin{array}{l}\text { Otros servicios excepto actividades del } \\
\text { gobierno }\end{array}$ \\
\hline 10 & Servicios financieros y de seguros & 20 & $\begin{array}{l}\text { Actividades del gobierno y de organismos } \\
\text { internacionales y extraterritoriales }\end{array}$ \\
\hline
\end{tabular}


El cuadro 2 muestra los resultados de los grados de densidad, cercanía e intermediación de la red económica del DF. Un hecho que destaca la integración de la economía mexicana: en los grados de densidad y cercanía tienen el mismo promedio, lo que significa que estas medidas están muy correlacionadas, además de que son conceptos parecidos. No obstante, la densidad y la cercanía hacen referencia a la integración, pero por un lado se actúa y por otro se interactúa. En el caso de la economía del DF el sector servicios es el más integrado en la estructura, tanto en la emisión como en la recepción de influencias. Sin embargo, para poder definir aquellos sectores que son altamente articuladores es necesario analizar estas tres medidas de forma simultánea. Es así que el sector electricidad, agua y suministro de gas por ductos al consumidor final, servicios educativos, otros servicios excepto actividades del gobierno y las actividades del gobierno y de organismos internacionales y extraterritoriales son los sectores más articuladores en la estructura económica del Distrito Federal.

\section{Cuadro 2}

Medidas de centralidad de la red económica del DF

\begin{tabular}{|c|c|c|c|c|c|}
\hline \multirow{2}{*}{$\begin{array}{c}\text { Número } \\
\text { de } \\
\text { sector }\end{array}$} & \multirow{2}{*}{ Sectores económicos } & \multicolumn{2}{|c|}{ Grado de densidad } & \multirow{2}{*}{$\begin{array}{c}\text { Grado } \\
\text { de } \\
\text { cercanía }\end{array}$} & \multirow{2}{*}{$\begin{array}{c}\text { Grado } \\
\text { de } \\
\text { intermediación }\end{array}$} \\
\hline & & Recepción & Emisión & & \\
\hline 1 & $\begin{array}{l}\text { Agricultura, ganadería, } \\
\text { aprovechamiento } \\
\text { forestal, pesca y caza }\end{array}$ & $20.0 \%$ & $10.0 \%$ & $15.0 \%$ & $1.8 \%$ \\
\hline 2 & Minería & $25.0 \%$ & $25.0 \%$ & $25.0 \%$ & $6.7 \%$ \\
\hline 3 & $\begin{array}{l}\text { Electricidad, agua y } \\
\text { suministro de gas por } \\
\text { ductos al consumidor } \\
\text { final }\end{array}$ & $55.0 \%$ & $15.0 \%$ & $35.0 \%$ & $8.2 \%$ \\
\hline 4 & Construcción & $5.0 \%$ & $25.0 \%$ & $15.0 \%$ & $1.2 \%$ \\
\hline 5 & $\begin{array}{l}\text { Industrias } \\
\text { manufactureras }\end{array}$ & $0.0 \%$ & $15.0 \%$ & $7.5 \%$ & $0.0 \%$ \\
\hline 6 & Comercio & $0.0 \%$ & $10.0 \%$ & $5.0 \%$ & $0.0 \%$ \\
\hline 7 & Transportes & $0.0 \%$ & $25.0 \%$ & $12.5 \%$ & $0.0 \%$ \\
\hline 8 & $\begin{array}{l}\text { Transportes y } \\
\text { almacenamiento }\end{array}$ & $10.0 \%$ & $15.0 \%$ & $12.5 \%$ & $1.5 \%$ \\
\hline
\end{tabular}




\begin{tabular}{|c|c|c|c|c|c|}
\hline 9 & $\begin{array}{l}\text { Información en } \\
\text { medios masivos }\end{array}$ & $5.0 \%$ & $15.0 \%$ & $10.0 \%$ & $0.9 \%$ \\
\hline 10 & $\begin{array}{l}\text { Servicios financieros y de } \\
\text { seguros }\end{array}$ & $0.0 \%$ & $15.0 \%$ & $7.5 \%$ & $0.0 \%$ \\
\hline 11 & $\begin{array}{l}\text { Servicios inmobiliarios } \\
\text { y de alquiler de bienes } \\
\text { muebles e intangibles }\end{array}$ & $10.0 \%$ & $20.0 \%$ & $15.0 \%$ & $2.0 \%$ \\
\hline 12 & $\begin{array}{l}\text { Servicios profesionales, } \\
\text { científicos y técnicos }\end{array}$ & $20.0 \%$ & $10.0 \%$ & $15.0 \%$ & $1.8 \%$ \\
\hline 13 & $\begin{array}{l}\text { Dirección de corporati- } \\
\text { vos y empresas }\end{array}$ & $20.0 \%$ & $10.0 \%$ & $15.0 \%$ & $2.3 \%$ \\
\hline 14 & $\begin{array}{l}\text { Servicios de apoyo a los } \\
\text { negocios y manejo de } \\
\text { desechos y servicios de } \\
\text { remediación }\end{array}$ & $20.0 \%$ & $10.0 \%$ & $15.0 \%$ & $1.8 \%$ \\
\hline 15 & Servicios educativos & $60.0 \%$ & $20.0 \%$ & $40.0 \%$ & $12.6 \%$ \\
\hline 16 & $\begin{array}{l}\text { Servicios de salud y de } \\
\text { asistencia social }\end{array}$ & $0.0 \%$ & $15.0 \%$ & $7.5 \%$ & $0.0 \%$ \\
\hline 17 & $\begin{array}{l}\text { Servicios de esparcimien- } \\
\text { to culturales y depor- } \\
\text { tivos, y otros servicios } \\
\text { recreativos }\end{array}$ & $0.0 \%$ & $15.0 \%$ & $7.5 \%$ & $0.0 \%$ \\
\hline 18 & $\begin{array}{l}\text { Servicios de alojamiento } \\
\text { temporal y de prepa- } \\
\text { ración de alimentos y } \\
\text { bebidas }\end{array}$ & $0.0 \%$ & $25.0 \%$ & $12.5 \%$ & $0.0 \%$ \\
\hline 19 & $\begin{array}{l}\text { Otros servicios excepto } \\
\text { actividades del gobierno }\end{array}$ & $50.0 \%$ & $25.0 \%$ & $37.5 \%$ & $12.3 \%$ \\
\hline 20 & $\begin{array}{l}\text { Actividades del gobierno } \\
\text { y de organismos interna- } \\
\text { cionales y extraterrito- } \\
\text { riales }\end{array}$ & $50.0 \%$ & $30.0 \%$ & $40.0 \%$ & $14.6 \%$ \\
\hline \multicolumn{6}{|c|}{ Fuente: Elaboración propia } \\
\hline
\end{tabular}

Como se aprecia, la forma de articulación es distinta en cada sector, sin embargo, desde la esfera productiva se orienta más a los servicios que al sector primario o secundario, pues en el caso de las manufacturas 
no tienen alto grado de intermediación, pero si de emisión de influencias como en el sector de servicios. Los efectos de un cambio en la forma de producir, expresado en un cambio en la proporción de insumos, se encadenan en mayor medida por los otros servicios excepto actividades del gobierno y por las actividades del gobierno y de organismos internacionales y extraterritoriales, ya que son los sectores que mayormente articulan la economía del DF.

\section{CONSIDERACIONES FINALES}

Este documento ha identificado la forma de articulación de los sectores económicos en el Distrito Federal a partir de la posición que guardan en la red económica construida por la posición de los llamados coeficientes importantes, es decir, los vínculos relevantes por donde se transmiten cambios de los coeficientes técnicos que favorecen en mayor medida el crecimiento del producto. Como muestran los datos, la economía del DF se fundamenta en el sector servicios, pero de estos destaca el de las actividades de gobierno, pues es de los sectores que más articula actividad económica.

A pesar de que la política mexicana es de no intervención económica, la influencia que tiene el Estado sobre la economía no puede neutralizarse. Este trabajo ayuda a reconsiderar que las políticas de gasto pueden exigir mejor calidad de servicios, como lo ha hecho con el transporte de la ciudad, y así identificar los encadenamientos del propio sector servicios, ya que aquí se ha identificado la articulación de la actividad económica, la senda de desarrollo que tiene la economía para los datos de 2008. Así, el instrumento del MIP ayuda a realizar un diagnóstico completo sobre la actividad económica del DF, que puede ser regionalizada o por sector, ya que se puede extender el análisis metodológico a nivel regional, en este caso para cada delegación en la entidad o para un análisis del flujo de transacciones interindustriales del sector servicios.

Otro aspecto a considerar es el papel del sector electricidad, agua y suministro de gas por ductos al consumidor final, ya que también es un agente central y articula la economía del DF. Adicionalmente, es un sector 
que a pesar de que está clasificado como sector que emplea tecnología media-alta, de acuerdo con la definición de la Organización de Cooperación y Desarrollo Económico, el peso del valor agregado de este sector es considerable sobre el producto, ya que por cada peso de producto 50 centavos son de valor agregado, de los cuales 13 centavos se destinan a las remuneraciones.

Al medir la articulación de los sectores económicos a partir de los conceptos propuestos de centralidad por la teoría de las redes sociales para el caso de la estructura económica del DF, ésta es articulada por las actividades del gobierno; electricidad, agua y suministro de gas por ductos al consumidor final; y por otras actividades de servicios excepto de gobierno, es decir, aquellas actividades que se dedican a reparación mecánica y eléctrica de autos y camiones, reparación y mantenimiento del hogar y del cuidado personal, los servicios sanitarios públicos, las asociaciones y organizaciones políticas, religiosas y civiles. Así estos tres sectores son actividades articuladoras que pertenecen a los servicios de la estructura de la entidad. 


\section{BIBLIOGRAFÍA}

Aroche, Fidel y M.A. Márquez. 2011. "Structural Integration, Exports and Growth in Mexico. An Input-Output Approach, Politic Economic Review, 24(1): 87 - 101.

Borgatti, S.P. 2005. "Centrality and Network Flow", Social Networks, 27(1): 55-71.

Cohen A.M. 1964. "Comunication Networks in Research and Training", Personnel Administration, 27: 18-24.

Freeman L.C. 1979. "Centrality in Social Networks Conceptual Clarification”, Social Networks, 1: 215-239.

García Muñiz, A. S., F. Aroche y C. Ramos. 2007. "Determinación de los Coeficientes Importantes por niveles tecnológicos", Investigaciones Económicas, XXXI(1): 161-190.

Hage P. y Harary, Frank. 1969. Exchange in Ocenia. A Graph Therretic analysis, Clarendon Press Oxford.

Hirschman, A. 1958. The Strategy of Economic Development, Yale University Press

Jiménez Reyes, Alonso. 2013. La estructura económica del Distrito Federal: un análisis mesoeconómico. tesis de maestria, borrador.

Krueger, A. 1990. Perspectives on Trade and Development, Chicago: University of Chicago Press

Leontief,, W. 1986. Input - Output Economics, 2da. ed., Oxford University Press.

Miller, R. y P. Blair. 2009. Input - Output Analysis: Foundations and Extensions, Cambridge University Press. 
Minian, Isaac y E. Dávalos. 2012. Redes globales y regionales de producción. México, IIEC-UNAM, CISAN-UNAM,

De Mesnard, L. 1995. "A Note on Qualitative Input - Output Analysis", Economic System Research, 7(4): 439-448.

Newman, M.E.J. 2005. "A Measure of Betweenness Centrality Based on Random Walks”, Social Networks, 27(1): 39-54.

Nieminen, J. 1973. "On the Centrality in Directed Graph”, Social Science Research, 2: 371-378.

Programa de Naciones Unidas para el Desarrollo(PNUD). 2010. Índice de desarrollo humano en México: cambios metodológicos e información para las entidades federativas. www.undp.org.mx/desarrollohumano

Ruiz Nápoles, P. y J.C. Moreno. 2006. "Efectos macroeconomicos de la apertura y el TLCAN" en M. Gambrill (comp.) Diez años del TLCAN en México, México, UNAM. pp. 19-34.

Shaw, M.E. 1954. "Group Structure and the Behavior of Individuals in Small Groups", Journal of Psychology, 38: 139-149.

Sherman, Jack y W.J. Morrison. 1949. “Adjustment of an Inverse Matrix Corresponding to Changes in the Elements of a Given Column or a Given Row of the Original Matrix (abstract)," Annals of Mathematical Statistics, 20: 621.

Schnabl, H. 1995. "The Subsystem-MFA: A Qualitative Method for Analyzing National Innovation Systems-The Case of Germany", Economic System Research, 7(4): 383-396.

Shintke, J. y R. Stäglin. 1988. "Important Input Coefficients in the Market Transactions' Tables and Production Flows Tables", en M. Ciaschini (comp.) Input-Output Analysis: Current Developments, Londres, Chapman and Hall. 